\title{
Three-dimensional force microscope: A nanometric optical tracking and magnetic manipulation system for the biomedical sciences
}

Cite as: Rev. Sci. Instrum. 76, 053711 (2005); https://doi.org/10.1063/1.1914777

Submitted: 18 November 2004 . Accepted: 24 March 2005. Published Online: 02 May 2005

J. K. Fisher, J. R. Cummings, K. V. Desai, L. Vicci, B. Wilde, K. Keller, C. Weigle, G. Bishop, R. M. Taylor, C. W. Davis, R. C. Boucher, E. Timothy O'Brien, and R. Superfine

Export citation

\section{ARTICLES YOU MAY BE INTERESTED IN}

Thin-foil magnetic force system for high-numerical-aperture microscopy

Review of Scientific Instruments 77, 023702 (2006); https://doi.org/10.1063/1.2166509

BaHigh-force magnetic tweezers with force feedback for biological applications

Review of Scientific Instruments 78, 114301 (2007); https://doi.org/10.1063/1.2804771

A magnetic manipulator for studying local rheology and micromechanical properties of biological systems

Review of Scientific Instruments 67, 818 (1996); https://doi.org/10.1063/1.1146816

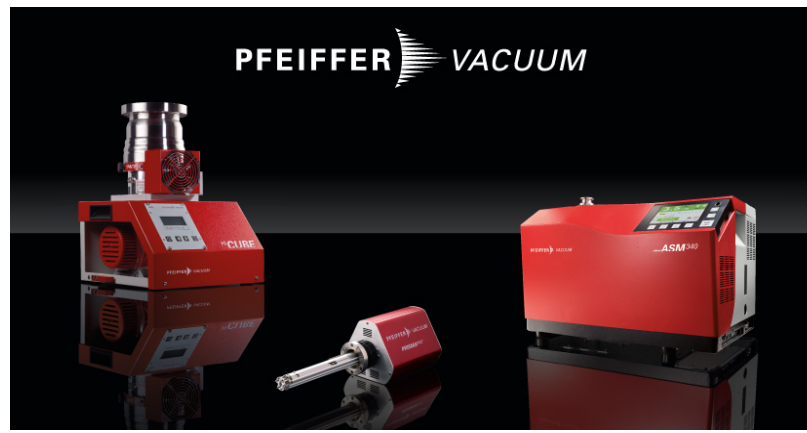

\section{Vacuum solutions from a single source}

Pfeiffer Vacuum stands for innovative and custom vacuum solutions worldwide, technological perfection, competent advice and reliable service.

Learn more! 


\title{
Three-dimensional force microscope: A nanometric optical tracking and magnetic manipulation system for the biomedical sciences
}

\author{
J. K. Fisher, J. R. Cummings, and K. V. Desai \\ Department of Biomedical Engineering, University of North Carolina, Chapel Hill, \\ North Carolina 27599-7575 \\ L. Vicci, B. Wilde, K. Keller, C. Weigle, G. Bishop, and R. M. Taylor II \\ Department of Computer Science, University of North Carolina, Chapel Hill, North Carolina 27599-3175 \\ C. W. Davis and R. C. Boucher \\ Medicine/Cystic Fibrosis Center, University of North Carolina, Chapel Hill, North Carolina 27599-7248 \\ E. Timothy O'Brien and R. Superfine \\ Department of Physics and Astronomy, University of North Carolina, Chapel Hill, \\ North Carolina 27599-3255
}

(Received 18 November 2004; accepted 24 March 2005; published online 2 May 2005)

\begin{abstract}
We report here the development of a three-dimensional (3D) magnetic force microscope for applying forces to and measuring responses of biological systems and materials. This instrument combines a conventional optical microscope with a free-floating or specifically bound magnetic bead used as a mechanical probe. Forces can be applied by the bead to microscopic structures of interest (specimens), while the reaction displacement of the bead is measured. This enables 3D mechanical manipulations and measurements to be performed on specimens in fluids. Force is generated by the magnetically permeable bead in reaction to fields produced by external electromagnets. The displacement is measured by interferometry using forward light scattered by the bead from a focused laser beam. The far-field interference pattern is imaged on a quadrant photodetector from which the 3D displacement can be computed over a limited range about the focal point. The bead and specimen are mounted on a 3D translation stage and feedback techniques are used to keep the bead within this limited range. We demonstrate the system with application to beads attached to cilia in human lung cell cultures. (c) 2005 American Institute of Physics.
\end{abstract}

[DOI: $10.1063 / 1.1914777$ ]

\section{INTRODUCTION}

Living organisms move. At the cellular level motility, cell division, and alteration of cell morphology and adhesion are produced by the forces generated by thousands of molecular motors and the assembly of protein polymers. ${ }^{1-5}$ Inside the cell, those forces produce active transport of vesicles and organelles, the movement of chromosomes during mitosis, and even replication, transcription, and translation of genetic information into proteins. ${ }^{6-12}$ Moreover, cells respond to both external forces and impose forces, for example, to produce macroscopic fluid flow in the airway. ${ }^{13}$ We report a new combination of methods we call the three-dimensional force microscope (3DFM) to measure and apply forces at the nanometer scale, and to assesd the response of specimens to the applied forces. These methods offer high temporal and spatial resolution with minimal interference due to probe attachment or specimen heating.

Numerous techniques have been developed to measure nanoscale cellular forces. Among the classical probe techniques, glass fibers or microneedles have been used to measure the effects of forces on the movement of chromosomes $^{14-16}$ and the force exerted by myosin on actin. ${ }^{17}$ More recently, atomic force microscopy, using techniques relying on the deformation of a cantilever spring element, ${ }^{18}$ has emerged as a suitable method to obtain subnanometer spatial resolution ${ }^{19}$ with picoNewton force sensitivity. ${ }^{20}$ Evans $^{21}$ has developed a method using a deformable vesicle attached to a pipette to measure the forces between membrane-bound molecules and target specimens such as other vesicles or flat substrates. While providing important insights within their domains, these methods suffer from the invasiveness of the attached fiber, cantilever, or pipette, as well as an inherent limitation in the directional flexibility of the probe.

To address these shortcomings, methods have been developed that use a refractive microbead, often below $1 \mu \mathrm{m}$ in diameter, as a mechanical probe. The bead can be free to move throughout the accessible volume within a specimen, or can be functionalized to be attached to specific molecular groups or proteins. Optical tweezers is a method that forms a trap using the power gradient of a focused laser beam to attract refractive materials toward the waist of the focus. ${ }^{22,23}$ The force generated on standard plastic microbeads by the optical trap can be varied by changing the intensity of the laser, and these forces can be accurately calibrated. ${ }^{24}$ Laser tweezers have been applied to a wide variety of biological problems, for example, measurements of the forces generated during DNA transcription, ${ }^{25}$ the properties of neuronal 
membranes, ${ }^{26}$ and the forces generated by the molecular motors dynein, kinesin, and myosin. ${ }^{27-31}$ Its limitations are in the achievable force (generally less than $200 \mathrm{pN}$ ), specimen heating at higher forces ${ }^{32,33}$ (approximately $10^{\circ} \mathrm{C} / \mathrm{W}$ of laser power at $1064 \mathrm{~nm}$ laser wavelength in water), and the nonspecificity of forces which act on all refracting particles and macromolecules within the range of the optical trap.

As with laser tweezers, magnetic methods apply a force to a particle through field gradients. In this case the field is magnetic and quite specific to a magnetically permeable bead because typical biological materials are at most only weakly magnetically active. Moreover, high magnetic fields at the frequencies of interest do not generate significant heat, so high forces can be applied to the bead without heating the specimen. A variety of magnetic force systems have been developed over the past 50 years beginning with the seminal work of Crick and Hughes ${ }^{34}$ in $1949 .{ }^{34,35}$ Many of the systems that have been reported apply forces in a single direction, usually with one pole tip. ${ }^{36-38}$ Valberg introduced a magnetic system designed for applying torques. ${ }^{39}$ Strick $e t$ $a l .{ }^{40}$ applied a multipole geometry to apply forces upward while applying a torque to a ferromagnetic bead. Amblard et $a l .^{41}$ introduced an eight pole device whose construction was designed to produce forces within the specimen plane. Following up on this, Huang et al. ${ }^{42}$ implemented a full octapole design. Gosse and Croguette presented a six pole design where the poles were located above the specimen with no magnetic forces possible in the downward direction. ${ }^{43}$ This system also included optical tracking of the bead through processing of images acquired by a camera. The magnetic force prototype we describe here uses four magnetic poles, which for design symmetry reasons are arranged in an equilateral tetrahedral geometry, which we demonstrate can produce forces in the nanonewton range, as well as forces in three dimensions.

In this article we describe the combination of this magnetic force system with a sensitive, high bandwidth 3D position tracking system. Our goal was to design an instrument in which the probe was free to travel over tens of microns while providing nanometer-scale position resolution. The position tracker uses an interferometric measurement technique capable of resolving nanometer scale 3D displacements in real time. ${ }^{44-46}$ The functional range of this tracking technique is limited to about a wavelength from the waist of a focused tracking laser beam. It is therefore necessary to be able to move the beam focus throughout the useful working volume of a specimen chamber, on the order of $50 \mu \mathrm{m}$ for many biological studies. To achieve this we used an $(x, y, z)$ translation stage to move the specimen chamber relative to the beam focus, which we fixed in laboratory coordinates.

With the addition of these three hardware components, we have augmented an optical microscope with the necessary 3D mechanical instrumentation to enable mechanical microscopy experiments in biological and other sciences. In addition to these basic instrumentation technologies, the 3DFM includes a feedback control system, a data acquisition system for time stamping and data logging, and a graphic user interface. Further, to allow the user to perform manipulations with natural hand motions, we have interfaced the system with a haptic force feedback device. ${ }^{47}$ The user can hold the force feedback pen in hand, and through natural hand motions control the position of the bead while feeling the reaction forces. In this article we focus on the hardware implementation and its application to the measurement of a challenging biological system.

\section{DESIGN GOALS}

The overall goal for our 3DFM was to be able to apply versatile, significant forces to microscopic structures while measuring reaction motions in $3 \mathrm{D}$. The major subsystems are comprised of an optical microscope, an interferometric position tracker, an active translation stage, a magnetic force generator, and a computer to integrate the subsystems. Each of the following specifications represents a compromise between scientific requirements and technical feasibility.

The optical microscope: A simple bright field configuration was deemed adequate for this prototype, and about a half micron resolution adequate for an interesting range of biological experiments. Over the visible spectrum, this is achievable with a 0.7 numerical aperture (NA) microscope objective. This moderate requirement allows use of long working distance lenses, easing the space constraints to accommodate the force and translation stage hardware.

Position tracking: Molecular motor experiments require resolution of a few nanometers at a bandwidth of at least $100 \mathrm{~Hz} .^{48}$ Passive measurement of real-time local viscoelastic properties benefits from a bandwidth of tens of kilohertz. ${ }^{49}$ Biological specimens such as cell cultures with beating cilia require a working volume of a few tens of microns in $z$, and perhaps $100 \mu \mathrm{m}$ in $x$ and $y$. Accordingly, the tracking system goal was to track a single particle with a resolution of $4 \mathrm{~nm}$ in $(x, y, z)$, in a working volume of at least $100 \times 100 \times 50 \mu \mathrm{m}^{3}$ at a bandwidth of $10 \mathrm{kHz}$. The interferometric tracking techniques developed for laser tweezers in principle can achieve these resolution and bandwidth requirements, but only in a volume of less than $1 \mu \mathrm{m}^{3}$. The addition of an active $(x, y, z)$ translation stage with feedback to keep the tracked bead within this volume enables tracking a single bead over the working volume of a specimen chamber, limited only by the range of the translation stage.

Force generation: Ultimately, the 3DFM should be applicable to an open ended set of experiments in biological and other sciences, just as for conventional microscopy. This makes force specification a rather nebulous exercise. For example, some experiments require maximum directional flexibility whereas others may need only to pull in one direction, but with high force. We therefore chose to aim for a system that could generate credibly high forces in particular directions in restricted parts of the working volume, and credibly general directionality at lower forces in other restricted parts. We also chose to accept a low force bandwidth to ease the magnet design. We aimed to demonstrate forces in the nanonewton range, bandwidths of a few tens of hertz, and force directionality over a substantial solid angle, symmetrically distributed in 3D. 


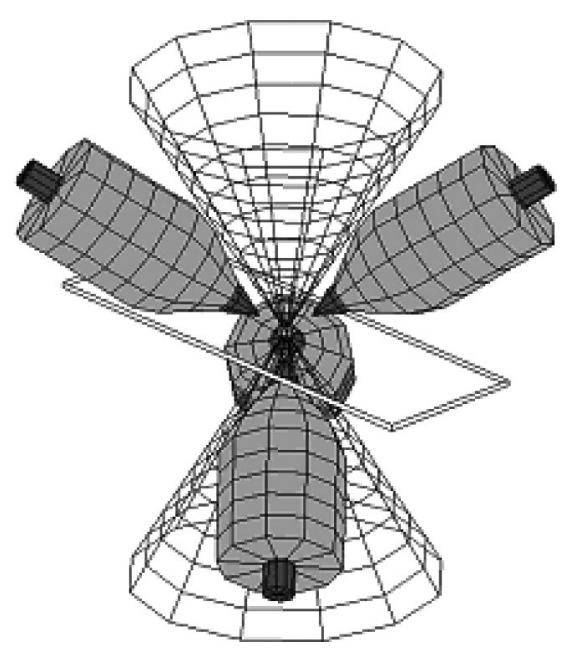

FIG. 1. Conceptual magnetic design with coils wound directly around poles, showing the tetrahedral pole geometry lying outside the numerical aperture of a reentrant optical path.

\section{THEORY AND DESIGN}

\section{A. System level}

Spatial coexistence is a major design challenge for the 3DFM. In order for the imaging, force, and tracking subsystems to simultaneously access the bead in the specimen chamber, they must coexist in space such that physical interference between them is kept within acceptable limits. The resource in question here is solid angle about the bead, which must be maintained at the tracking beam focus which itself must be in the specimen chamber. The specimen chamber comprises two closely spaced, parallel, optical quality cover slips with means for sealing a liquid specimen between them. The imaging and tracking subsystems share the same optical path near the specimen, both traveling through the objective and condenser. We use identical condensing and objective lenses, arranged in a reentrant confocal configuration, with dichroic beamsplitters allowing for the separation of the tracking laser from the imaging beam path. The tracking system uses coherent near infrared laser light, whereas the imaging system uses the visible spectrum. For NA $=0.7$ lenses, the optical path occupies a pair of reentrant cones with included vertex angles of about $89^{\circ}$, symmetrically disposed about the optical axis. The magnetic system and translation stage hardware must fit into the remaining solid angle. Implementation details are discussed in subsequent sections.

\section{B. The force subsystem}

The force subsystem comprises the force probe consisting of a magnetic bead, and multiple electromagnets arranged in space to enable forces to be applied to the bead in 3D. Shaped magnetic cores are used to conduct the magnetic flux from the bulky coils of the electromagnets to the specimen space, as shown in a concept sketch, Fig. 1. The cores are tapered to concentrate the flux, ending in sharpened pole tips. Flux leaving the pole tips will naturally diverge, mostly entering other poles. The bead will always be attracted in the direction of increasing field gradient, so it is possible to pull a bead towards any pole tip, but never to push it away. To apply $3 \mathrm{D}$ forces to the bead requires at minimum four noncoplanar pole tips.

The most symmetric arrangement for four poles is in an equilateral tetrahedral geometry, as shown in Fig. 1. By suitable choice of orientation, the four poles can be located with their axes angularly equidistant from the optical axis. This optimizes angular clearance between the poles and the optical path. The included angle between any two vertices of a tetrahedron is about $109^{\circ}$, whereas the included angle of the optical path is about $89^{\circ}$, giving an angular clearance of $10^{\circ}$ between the axes of the poles and the optical path.

\section{Magnetic force equations}

Force on a magnetic bead is caused by an interaction between its magnetic dipole moment $\mathbf{m}$ and the gradient $\nabla \mathbf{B}$ of an incident magnetic field. For a soft, magnetically permeable bead, $\mathbf{m}$ is entirely induced by the incident field. Subject to saturation properties of the magnetic material in the bead,

$$
\mathbf{m}=\frac{\pi d^{3}}{2 \mu_{0}}\left(\frac{\mu_{r}-1}{\mu_{r}+2}\right) \mathbf{B}
$$

where $\mu_{0}$ is the permeability of free space in SI units, $\mu_{r}$ is the relative permeability of the bead, and $d$ is the diameter of the bead. The potential $U$ of a paramagnetic bead is given by $U=\frac{1}{2} \mathbf{m} \cdot \mathbf{B}$, with the corresponding magnetic force (the gradient of the potential) being

$$
\mathbf{F}=\frac{\pi d^{3}}{4 \mu_{0}}\left(\frac{\mu_{r}-1}{\mu_{r}+2}\right) \nabla\left(B^{2}\right) .
$$

To estimate $\mathbf{B}$ in the specimen chamber, the pole tips can be reasonably well modeled by magnetic monopoles, provided that the excitations are properly constrained such that the aggregate generated pole flux sums to zero. Otherwise a return path must be modeled for the non-zero residual flux, because there are in nature no actual sources or sinks of magnetic flux (i.e., no monopoles). This return path could be modeled with a multipole expansion on a boundary enclosing the poles, but we chose the simpler model and, as will be described, the as-built design closely approximates the zero net flux constraint.

\section{Bead force considerations}

According to the monopole model, the magnitude of $B$ from a singly excited magnetic pole is proportional to $B_{p} / r^{2}$, where $B_{p}$ is the pole strength and $r$ is the distance from the pole. Correspondingly, $\nabla\left(B^{2}\right)=-4 B_{p}^{2} r^{5}$, directed toward the pole. Clearly, the distance between a pole tip and the bead is of primary importance in optimizing bead force.

Optimizing $B_{p}$ is also important. The $B_{p}$ of each pole is proportional to its coil current. For a given current, the coil dissipates heat proportional to its resistance, which is inversely proportional to its volume. Thus, large coils are needed to obtain high $B_{p}$ without excessive heating. $B_{p}$ is also inversely proportional to magnetic path reluctance $R$, which is the sum of the reluctances $R_{i}$ of the constituent pieces of the total magnetic path, each of which can be approximated as $R_{i} \approx l_{i} / \mu_{r}^{i} A_{i}$ where $l_{i}$ is the length and $A_{i}$ the 
cross-sectional area of piece $i$, and $\mu_{r}^{i}$ is its relative magnetic permeability. A simplified model of the magnetic path for each pole consists of the pole itself, a gap through the specimen space, the parallel combination of the other three poles, and the return path between the back sides of all four poles. Magnetic path materials are commonly available having $\mu_{r}$ $>5000$. Consequently, the path reluctance is dominated by the gaps in the circuit. To provide a good magnetic return path, a high $\mu_{r}$ magnetic shell can be added to the configuration shown in Fig. 1 to connect the back sides of the poles. ${ }^{42}$ The gradient $\nabla B$ is predominantly affected by pole tip geometry. To maximize gradient, the tips should be sharp, and located close to the specimen.

\section{Mechanical trade-offs}

We used 0.7 NA Mitutoyo APO 100 lenses (Mitutoyo America Corporation, Aurora, IL), chosen for their generous $6 \mathrm{~mm}$ working distance. The lens bodies are wider than the optical path itself and fill an angle of $110^{\circ}$, which is very close to the $109^{\circ}$ included angle between tetrahedral pole axes. The finite diameters of the magnetic poles precludes arranging their axes in equilateral tetrahedral angles, unless the lens bodies or the poles themselves are modified with relief cuts. A simpler solution was to modify the pole axis angles to $135^{\circ}$ (or from $35.3^{\circ}$ to $22.5^{\circ}$ inclination from horizontal), while preserving the tetrahedral pole tip positions. A pole tip taper angle of $9.5^{\circ}$ leaves $7.75^{\circ}$ angular clearance from the lens body. However, this clearance is diminished as the size of the tetrahedral working volume is increased to accommodate a usable specimen space.

The pole tips are of course not infinitely sharp points, however they may be regarded as spherical. For high permeability core material, the modeled magnetic monopoles are located approximately at the centers of these spheres. Thus, for a specimen chamber consisting of two horizontal cover slips enclosing a liquid cell, the vertical separation between the upper and lower monopole locations is the sum of the sphere radii $(\leqslant 50 \mu \mathrm{m})$, the specimen chamber thickness $(\cong 400 \mu \mathrm{m})$, and $100 \mu \mathrm{m}$ working clearance between the pole tips and the specimen chamber. This comes to $600 \mu \mathrm{m}$ of vertical separation. The total distance from each monopole to the center of the specimen chamber is therefore $(600 / 2) / \cos (109 / 2)=517 \mu \mathrm{m}$. For this specimen chamber size, $517 \mu \mathrm{m}$ is the closest we can reasonably expect to get the magnetic poles in this design.

Referring to Fig. 1, it is evident that the coils are awkwardly placed. Not only must they be tapered in the front, but it would be difficult to fit them inside a magnetic shell that would be necessary to minimize the return path reluctance. This problem, along with the need for a large number of ampere turns in a limited conductor cross section, and the fact that winding coils with a precision taper is difficult, led us to reconsider this seemingly straight forward design. The solution was to reposition the coils to locations sufficiently removed from the optical path that they could be made larger without interference. To do this, the return path geometry was configured as a frame with coils encircling parts of the return path rather than the poles themselves, as shown in Fig. 2.

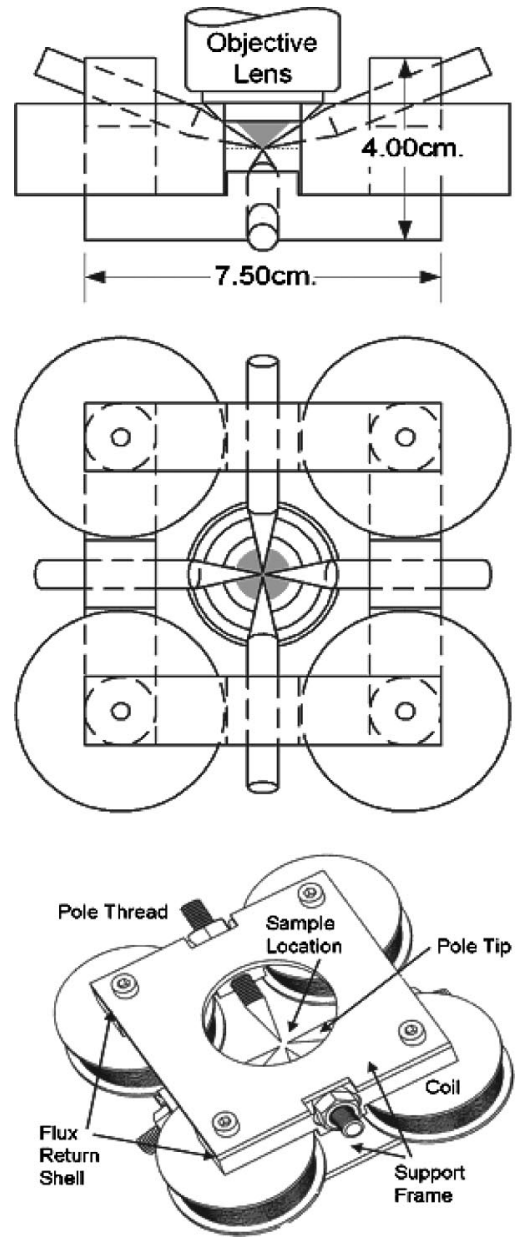

FIG. 2. Engineering drawings of the as-built design, showing the four magnetic poles and four coils: side view, bottom-up view and perspective view, respectively. One of two objective lenses is also shown in the first two views.

To allow for a large number of ampere turns, the coils were wound on cylindrical bobbins with a generous crosssectional area, an inner diameter of $1.7 \mathrm{~cm}$, an outer diameter of $4.25 \mathrm{~cm}$, and a length of $2.0 \mathrm{~cm}$. They consisted of 340 turns of \#22AWG Belden \#8077 (Belden Division, Richmond, IN) magnet wire. The temperature rise of the coils driven at $3 \mathrm{~A}$ for $3 \mathrm{~min}$ was $22.7^{\circ} \mathrm{C}$. This provides coils capable of generating a magnetomotive force (MMF) greater than 1000 ampere turns.

\section{Magnetic circuit topology}

Relocating the coils represents a change in the magnetic circuit toplogy such that the MMFs drive the return path legs of the circuit. Schematic diagrams of electrical analogs to the magnetic circuits are shown in Fig. 3. The MMFs are represented by voltage sources, and the reluctances of the various pieces of the magnetic path are represented by resistors.

Figure 3(a) shows the topology of the conceptual design of Fig. 1 with a magnetic return path shell added. A coil encircling each pole provides a MMF $M_{i}$ in series with pole reluctance $R_{p}$ and specimen reluctance $R_{a}$ due to the air/ glass/liquid gap between the pole tip and the location of the 

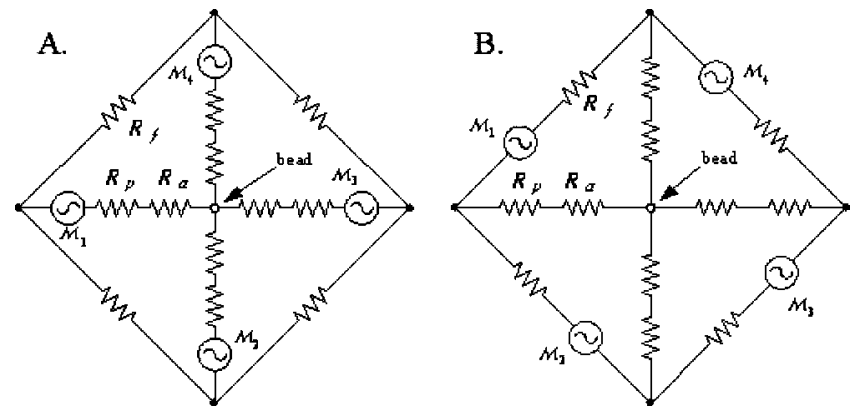

FIG. 3. Schematics of electric circuit analogs to the magnetic paths. The reluctances $R$ represent the flux return leg reluctance $R_{f}$, the pole tip reluctance $R_{p}$, and the reluctance $R_{a}$ of the gap between the pole tip and the bead. The magnetomotive forces $M_{i}$ are generated by currents in coils wound around their respective legs. For clarity, labels of symmetrically defined reluctances have been omitted. (A) Electric circuit analog of the conceptual design with the coils wrapped around each pole tip, whereas (B) is for the as-built design where the coils wrap around the flux return path segments.

bead. This is conceptually straightforward, where the $B_{p}$ of each pole is proportional to its coil current, with regard to magnetic saturation of the pole.

Figure 3(b) shows the as-built topology. In this case, four coils encircle four return path pieces which connect the back ends of the pole cores in a ring. These are shown as MMFs $M_{i}$ in series with their respective return path circuit reluctances $R_{f}$. Pole reluctances $R_{p}$ connect the junctions between the $R_{f}$ to edges of the specimen space, and specimen reluctances $R_{a}$ connect their respective pole tips to the bead.

In both cases, the specimen space reluctances dominate. It should be appreciated that these are simplified models, and that parasitic reluctances actually exist between every pair of circuit nodes, some of them being significant. They are omitted here for clarity in understanding the behavior of the asbuilt topology.

Referring to Fig. 3(b), we note that the $R_{f} \ll R_{a}$ which ensures that for any possible excitation of coils, any tendency to produce a flux imbalance in the poles will instead be shunted through the return path, thereby satisfying the flux rule required for the monopole model to work. We also note that to drive any single pole requires exciting both coils adjacent to it, but in opposite directions. This produces two MMFs in parallel to drive the pole. The resulting flux passes through the pole and specimen space, distributes itself according to the reluctances $R_{a}^{i}$, and returns through the other three poles. If good symmetry is achieved in the as-built design, the path reluctances $R_{a}^{i}$ will all be equal and the flux will split equally three ways. For a bead at the center of the specimen chamber, this achieves about $90 \%$ of the theoretically maximum force obtainable by an optimal (but less symmetric) pole tip placement.

\section{Core design}

Any gap in a high $\mu_{r}$ magnetic core will strongly affect the $B$ field induced by a given MMF. For example, in a $3 \mathrm{~cm}$ diameter toroidal core with $\mu_{r}=1000$, introducing a $100 \mu \mathrm{m}$ air gap where $\mu_{r}=1$ will approximately halve the induced $B$ field. This sensitivity makes the specimen space gaps critical to performance in two respects. First, is a trade off between specimen chamber size and required MMF (and hence coil current). The other is uniformity of gap size between poles. This also is a compelling reason to avoid as many other gaps in the magnetic paths as possible.

The as-built design is shown in Fig. 2. The return path topology is a ring, geometrically implemented as four cylindrical coil cores between two rectangular upper cross pieces and two lower ones arranged crosswise to the upper ones. This assembly is held together by four screws through the cross pieces and the centers of the coil cores so that all mating surfaces are under compression to avoid significant gaps. The screw holes are oversized sufficiently to provide tolerance for adjustment of the $x$ and $y$ pole tip positions during assembly. The cross pieces have threaded holes drilled at the pole axis inclination angles of $22.5^{\circ}$, into which the threaded poles are screwed and secured with jam nuts. This provides assembly-time pole tip position adjustability in $z$. After assembly, the entire magnetic unit is rigid and robust to misalignment during operation.

Due to the tapered geometry of the pole tips, the flux in the pole becomes more concentrated as it approaches the tip. As the MMF is increased, a threshold is reached where the pole saturates just at the tip where it is narrowest. A further increase causes the saturation point to move backward from the tip toward the root of the pole. Any flux in excess of saturation behaves as if the pole $\mu_{r}=1$, and is not guided by the pole, rather it is free to leak away through the sides of the taper. Consequently, the maximum obtainable $B_{p}$ at the pole tip is the saturation induction $B_{\text {sat }}$ of the pole material, irrespective of the MMF. Thus, for a given tip geometry, the maximum attainable force depends quadratically on the saturation of the core material.

The highest saturation materials available are permendur $(2.45 \mathrm{~T})$ and hiperco $(2.42 \mathrm{~T})$, both of which are relatively expensive, and difficult to fabricate. A good compromise (and our choice of material) is ASTM A 848 Magnet Iron, which saturates at about 2.15 T (ASTM Standard Designation A 848/A 848M-96). ${ }^{50}$

These materials are all electrically conductive metals. According to Faraday's law $\nabla \times \boldsymbol{E}=-\partial \boldsymbol{B} / \partial t$, voltages are generated in the core by any time variation of $\boldsymbol{B}$. This in turn causes eddy currents to circulate in the cores, which limits the useful frequency range over which we can control $\boldsymbol{B}$. We chose not to complicate the design by lamination or other such techniques, and settled for the consequential low bandwidth, measured to be about $20 \mathrm{~Hz}$.

\section{Electronics}

The relevant drive parameter for the coils is current. Since the coils are inductive, and their dc resistances vary with temperature, controlling coil voltage will not accurately control $B_{p}$, or the resulting force. Therefore four power transconductance amplifiers were used, providing coil currents proportional to their input voltages. ${ }^{51}$ These were driven by the outputs of a data acquisition system interfaced to the control computer. The transconductance amplifiers were built from National Semiconductor LM675T power op amps. A schematic of the drive amplifier circuit is shown in Fig. 4. 


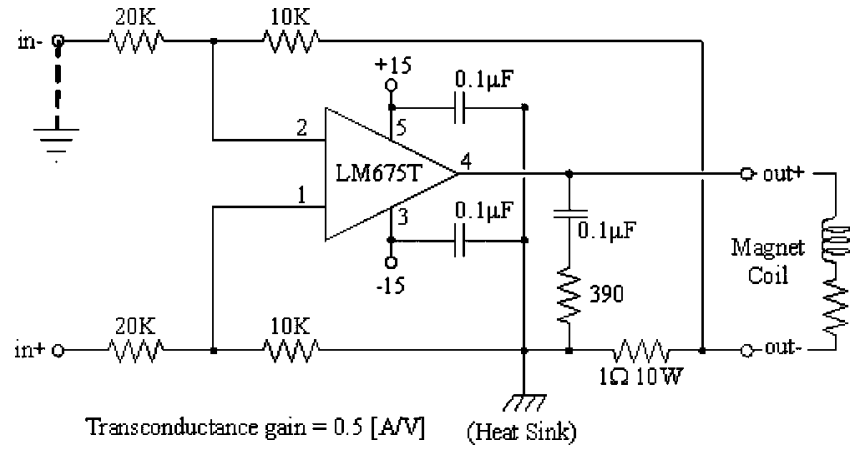

FIG. 4. Electrical schematic of a transconductance coil driver amplifier.

\section{The tracking subsystem}

We used a forward light scattering technique ${ }^{46,52}$ with little modification. We describe here the general principles, but refer readers to the references for details. This technique has the ability to track particles in full 3D at rates limited only by the photodetectors, far in excess of the capabilities of video tracking. By itself however, its usable working volume is only on the order of a wavelength of the light used. To be useful for the 3DFM, we must be able to dynamically reposition this working volume anywhere within the $100 \mu \mathrm{m}$ extent of the 3DFM specimen chamber. This capability is provided by adding an active positioning stage driven by the control computer, and using active feedback to keep the particle within the optical tracker working volume. The remainder of this section discusses the behavior of the optical tracker only within its own working volume.

\section{Interferometric tracking}

The physical principle used is interferometry, which requires a sufficiently coherent light source, for which we use a near infrared laser diode. The tracker uses the interference between a reference beam and a measurement beam to produce a diffraction pattern which represents the magnitude and phase of the measurement beam in a detection plane.

In the method by Pralle et al. $;^{46}$ the laser beam is focused by a condenser lens to a diffraction limited beam waist. An objective lens is placed on the opposite side and focused on the waist to capture the exiting beam. This reference beam is in the shape of two reentrant cones between the confocally arranged lenses. In the far field, the reference beam behaves as if it were emitted from a point source located at the beam waist.

If a small sphere (bead) with index of refraction different from its surrounding medium is placed within the reference beam, it will scatter some fraction of the beam. The scattering pattern can be quite complicated, but for beads less than half a wavelength in diameter, only the principal forward scattered lobe lies within the solid angle of the reference beam. The scattered light from the bead can be approximately regarded as originating from an isotropic point source located at the center of the bead. Larger beads also behave as nearly point sources, but with nonisotropic radiation patterns, a detail we shall not discuss here. In the far field, the interference of these two beams carries 3D position information in the form of a diffraction pattern.
A property of an ideal lens is that the light fields at the front and back focal planes of the lens are Fourier transforms of each other. Accordingly, the diffraction pattern at the back focus of the objective lens is a Fourier transform containing bead displacement information. A relay lens is used to reimage the back focal plane of the objective lens onto a quadrant photodiode (QPD) to capture the information in this pattern.

A bead exactly at the beam waist will scatter a measurement beam in some fixed phase relative to the reference beam. In the far field, the phase difference between the beams is uniform over angle, so the interference pattern is featureless. If the bead is displaced in $x$, a fringe pattern with an $x$ dependence will occur; similarly for $y$.

Measuring $z$ displacement relies on a phenomenon near the beam waist known as the Gouy phase shift in which light in the near field about the beam waist propagates at apparently superluminal speed. Intuitively, this has to happen for the concave spherical wavefronts entering the beam waist zone to deform to planar at the waist, then to convex as they leave the zone.

If the bead is displaced from the beam waist in $z$, it will scatter a measurement beam in some fixed phase relative to the Gouy-shift dependent phase of the incident beam. This $z$-dependent phase variation is propagated to the far field where it interferes with the fixed phase of the reference beam. To be sure, a distance dependent "bull's eye" diffraction pattern occurs anyway, but is too subtle to be of much use by itself. The Gouy phase shift causes a local but marked variation in intensity of the center of the bull's eye with $z$ displacement of the bead.

\section{Implementation}

Ideally the sum of all four QPD signals is a function of $z$ displacement, while the difference between the signals from the $-x$ and $+x$ sides of the QPD is a function of $x$ displacement, and similarly for $y$. Accordingly, we measure the four QPD signals $q_{i}$ from which we calculate the three functions

$$
\begin{aligned}
& X=\left(q_{0}+q_{2}\right)-\left(q_{1}+q_{3}\right), \\
& Y=\left(q_{0}+q_{1}\right)-\left(q_{2}+q_{3}\right), \\
& Z=q_{0}+q_{1}+q_{2}+q_{3} .
\end{aligned}
$$

The dependence of $(X, Y, Z)$ on the bead displacement $(x, y, z)$ is nonlinear; indeed even nonmonotonic. However sufficiently close to the beam waist, linearized "sweet spots" exist in which the bead can be maintained by feedback to the translation stage. The precisely measurable movements of the stage are then representative of the position of the bead relative to the specimen coordinates which are fixed to the translation stage.

\section{The imaging subsystem}

We have added the magnetic force and optical tracking subsystems to a custom built optical microscope subsystem, as shown schematically in Fig. 5. It was built for brightfield microscopy with Koehler transmission illumination. The microscope is mounted on a $1.219 \times 3.048 \mathrm{~m}$ vibration isola- 


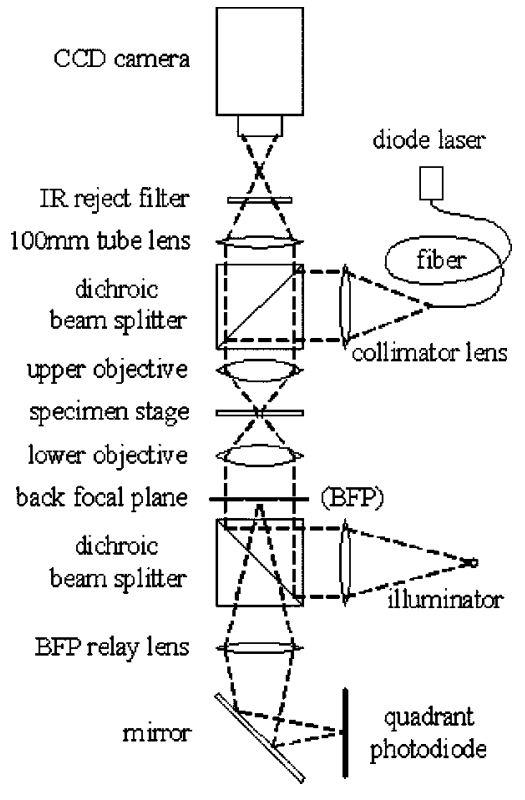

FIG. 5. Schematic of optical layout. The laser tracking system consists of a diode laser and a quadrant photodiode which are added to an otherwise standard transmission brightfield microscope using dichroic beam splitters. The near IR laser tracking light propagates in the opposite direction from the imaging system's visible light.

tion table (Newport, RS4000-410-12, Irvine, CA). An optical fiber couples a diode laser to the upper part of the setup where the condenser is located. The fiber coupling allows for dynamic translation of the setup without realignment of the laser optics. The laser beam diverging from the optical fiber is collimated using a lens with a focal length of $15.36 \mathrm{~mm}$ (Thorlabs, C260TM-B, Newton, NJ). This collimated beam has a measured beam waist diameter of $2.18 \mathrm{~mm}$. The laser light power is $2.28 \mathrm{~mW}$ at the entrance pupil of the upper objective. The (identical) objective and condenser lenses used are Mitutoyo M Plan Apochomatic $100 \times$ with a working distance of $6 \mathrm{~mm}$ and a NA=0.7. The long working distance objectives were chosen in order to accommodate the magnetic poles, at the expense of numerical aperture and therefore spatial resolution.

We chose a diode laser at $785 \mathrm{~nm}$ wavelength for stability $[$ root mean square $(\mathrm{rms})=0.03 \%>30 \mathrm{~Hz}$ ] and for a wavelength that would not interfere with fluorescence microscopy intended to be incorporated later. The laser (Thorlabs Inc. LPS-4224-785-FC, Newton, NJ) was driven by a feedback stabilized diode controller (Thorlabs Inc. LDC2000) and temperature controller (Thorlabs Inc. TEC2000, Newton, NJ) Power fluctuations from a HeNe laser were determined to be unacceptable for the purposes of tracking $(\mathrm{rms}=0.5 \%>30 \mathrm{~Hz})$.

The imaging subsystem accommodates a specimen chamber consisting of two cover slips separated and sealed by traditional adhesive methods. The spacing between the two coverslips ranges between 100 and $170 \mu \mathrm{m}$, and the specimen chamber volume holds approximately $15 \mu \mathrm{l}$ of fluid. The specimen chamber is mounted on a custom machined cantilevered specimen holder which includes two z-piezos (Thorlabs, AE0505D16, Newton, NJ) for nanometer resolution $z$ translation. The specimen holder is attached to a closed loop $x-y$ piezo translation stage (JDS Uniphase, Queensgate NPS-XY-100A, East Meadow, NY) for accurate nanometer positioning of the specimen. The $x-y$ piezo translation stage is mounted on a manual xyz-translation stage (Newport, model 401 and 423, Irvine, CA) for macroscopic positioning.

\section{E. The computer control and data acquisition subsystem}

The magnetic coils are driven in an open-loop configuration by a computer controlled D/A board (National Instruments, PCI-6713, Austin, TX). The output from this board is bipolar between -10 and $+10 \mathrm{~V}$ but due to the limitations of our transconductance amplifiers ( $\sim 3 \mathrm{~A}$ maximum output) we limit the drive signal to $\pm 6 \mathrm{~V}$. The four $\mathrm{D} / \mathrm{A}$ outputs are controlled by a program written in Microsoft Visual $\mathrm{C}++$. The program can generate combinations of arbitrary currents in the coils or can be programmed to generate fixed currents over arbitrary time periods so that the four magnetic poles are time multiplexed to apply a piecewise linear 3D force trajectory to the magnetic bead. In addition, the bead can be manipulated "by hand" using a 3D force feedback joystick (SensAble Technologies, Woburn, MA). The optical signal from the QPD is amplified by four transimpedance amplifiers with a gain of $26.7 \mathrm{~V} / \mathrm{mA}$. The amplifiers provide low pass filtering with a cutoff frequency $(-3 \mathrm{~dB}$ bandwidth) of $63 \mathrm{kHz}$. Each of the four channels is sampled at $25 \mathrm{kHz}$ (National Instruments, PCI-MIO-16XE, Austin, TX) which results in significant aliasing of high frequency noise $(\sim 10 \times$ our theoretical noise floor limit), this aliasing will eventually be corrected through the use of a faster A/D board. The A/D and D/A boards and software run on a Dell Precision 410 with dual $400 \mathrm{MHz}$ processors and $512 \mathrm{Mb}$ RAM.

\section{RESULTS AND DISCUSSION}

\section{A. Tracking resolution and noise measurements}

We tested the performance of the optical tracking subsystem using Polysciences, 0.472 $\pm 0.013,0.752 \pm 0.021$, $0.957 \pm 0.013 \mu \mathrm{m}$ polystyrene beads (Polysciences, Inc., Warrington, PA), and Estapor MC05N $940 \mathrm{~nm}$ paramagnetic beads (Fontenay Sous Bois, France). In practice, the $z$ sensitivity is relatively modest, $\sim 13 \%$ of the average QPD current level. For a $957 \mathrm{~nm}$ polystyrene bead, a $z$ displacement of $1 \mu \mathrm{m}$ gives a $Z$ variation of $\sim 80 \mathrm{mV}$ on a dc offset of $\sim 600 \mathrm{mV}$. The differential nature of $X$ and $Y$ provides better sensitivity. An $x$ or $y$ displacement of $1 \mu \mathrm{m}$ gives a peak-topeak signal of $\sim 150 \mathrm{mV}$ with a typical dc offset close to zero.

Pralle et al. ${ }^{46}$ reported that at displacements $>1 \mu \mathrm{m}$ there is significant cross talk between the lateral and axial displacement signals of the particle. Moreover, as verified in practice, the functions $X, Y$, and $Z$ are not linear over the tracker working volume. Therefore, accurate particle tracking requires a tight feedback loop to keep the particle as close as possible to the tracker's "sweet spot," near the center of the beam waist. 


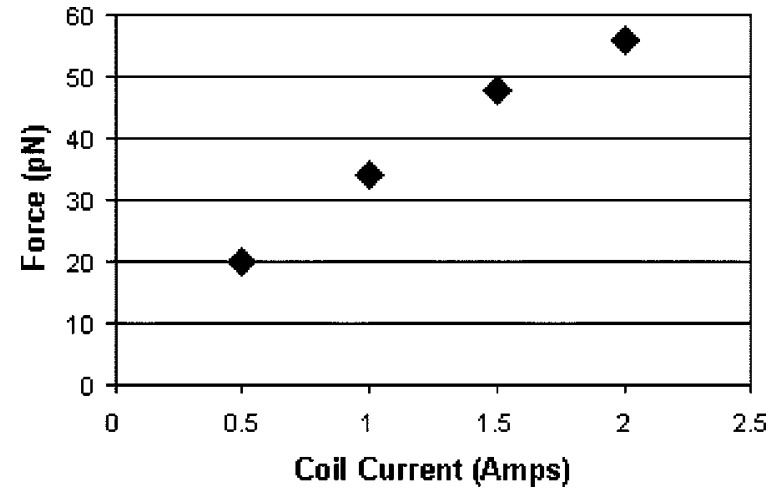

FIG. 6. Forces generated on $4.4 \mu \mathrm{m}$ beads at the center of the tetrahedral geometry, approximately $500 \mu \mathrm{m}$ from each pole tip. Coils were excited to generate a positive flux through one pole tip, returning through the remaining three. Data were taken in a fluid viscosity of $86 \mathrm{cP}$.

The total noise of the tracking subsystem was measured by fixing the beads to a coverslip and observing the measurement fluctuations of the position of the fixed bead. Fixation was performed by allowing the beads to dry out on the bottom coverslip and then rehydrating them before closing the specimen chamber. Upon rehydration only a small percentage $(<10 \%)$ were not completely immobilized and only those beads that appeared completely immobilized were chosen for measurements. The system noise floor, measured while actively tracking a fixed $752 \mathrm{~nm}$ polystyrene bead, was $3.1 \mathrm{~nm}$ RMS in $x$ and $y$, and $7.0 \mathrm{~nm}$ in $z$, with a bandwidth of about $200 \mathrm{~Hz}$ and $1.36 \mathrm{~mW}$ optical power at the specimen (1.36 $\mathrm{mW}$ corresponds to a specimen temperature rise of less than $\left.0.014{ }^{\circ} \mathrm{C}\right) .{ }^{32,33}$

\section{B. Magnetic force measurements}

The magnetic forces were determined by measuring the velocity of the beads in fluids of known viscosity, including a neutrally buoyant solution of corn syrup. The viscosity of the corn syrup was measured as $1.31 \mathrm{~Pa} \mathrm{~s}$ at $25^{\circ} \mathrm{C}$ using a commercial viscometer (Cannon Fenske, Viscometer Type No.513, State College, PA). The magnetic forces were calculated from Stokes formula, $F=6 \pi \eta a_{b} v$, where $\eta$ is the fluid viscosity, $a_{b}$ is the bead radius, and $v$ the bead velocity. In maximum force experiments, video data taken at 30 frames per second was tracked to determine particle velocities for beads close to a pole tip. For these experiments, symmetry was sacrificed and the pole was allowed to enter the specimen chamber. Close to a pole tip we measured forces as large as $200 \rho \mathrm{N}$ on $0.94 \mu \mathrm{m}$ beads, $4 \eta \mathrm{N}$ on $2.8 \mu \mathrm{m}$ beads, and $9 \eta \mathrm{N}$ on $4.4 \mu \mathrm{m}$ beads. Examples of experiments that require these higher forces and have used large beads include measurements of the mechanical properties of cell membranes ${ }^{53}$ and investigations of interacellular signals as a response to mechanical stresses. ${ }^{54}$ Other experiments under consideration in our lab that would require larger forces include measurements of forces involved during the different stages of cell division. ${ }^{14}$ Forces measured in the center of the specimen chamber were considerably smaller, but directionally far more symmetric. Fig. 6 shows the magnetic force variation with applied current, and Fig. 7 shows variation with distance from the pole tip.

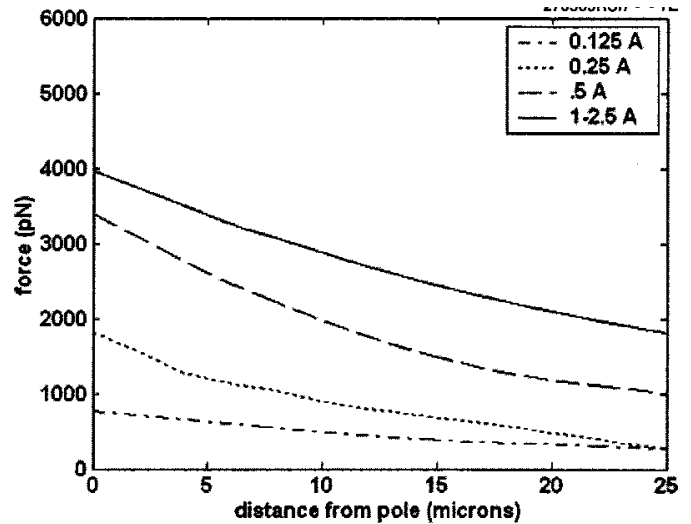

FIG. 7. Forces generated on a $2.8 \mu \mathrm{m}$ paramagnetic bead located near one of the pole tips. Forces as large as $4 \mathrm{nN}$ were achieved.

\section{Application to measurement of cilia function}

Cilia are ubiquitous actuating structures in biology, present in unicellular, simple multicellular and complex multicellular organisms including vertebrates. ${ }^{55}$ The rapid oscillation or "beating" of cilia is used for locomotion and translocation of food particles by unicellular ciliates such as paramecia. It is also used for transporting food particles and fluids by stationary multicellular organisms such as tunicates. ${ }^{55}$ Of the many functions served by cilia in humans, we are studying their role in the clearance of infectious agents and particulates from the lung.

The alveolar surface of the human lung is bathed in a thin aqueous layer covered by a thicker and more complex layer of mucus. Mucus is a nonuniform viscoelastic fluid composed of mucin proteins, glycosaminoglycans, and cellular remnants such as actin and DNA. The mucus layer sticks to and traps particulates, bacteria, and other infectious agents. The beating cilia propel this layer out through the airways toward the throat, ${ }^{56}$ where the mucus and its entrained detritus are swallowed and disposed of safely in the gut. A basic understanding of this process and how it can fail is fundamental to several important research areas: environmental factors affecting lung function, developing new drug delivery methods, studying the underlying causes of such diseases as cystic fibrosis, ${ }^{57}$ and developing new treatments for them.

The pattern of the ciliary beat, and the force applied by the forward, or "power" stroke of the cilia tips as they engage the mucus, are essential to understanding how the cilia propel mucus. Previous experiments studying the beat pattern have employed optical microscopy and high speed video imaging, ${ }^{58-61}$ both inherently two-dimensional instruments. The measurements of forces exerted by cilia have heretofore been limited to the compound cilium of single cell organisms ${ }^{62}$ and to the flagella of bull sperm. ${ }^{15}$ In this study, we used the 3DFM to track the motion of cilia in living human lung cell cultures and to explore their response to applied forces.

\section{Methods}

For cilia preparations, human airway epithelial cell cultures were obtained and grown on T-COL membrane sup- 


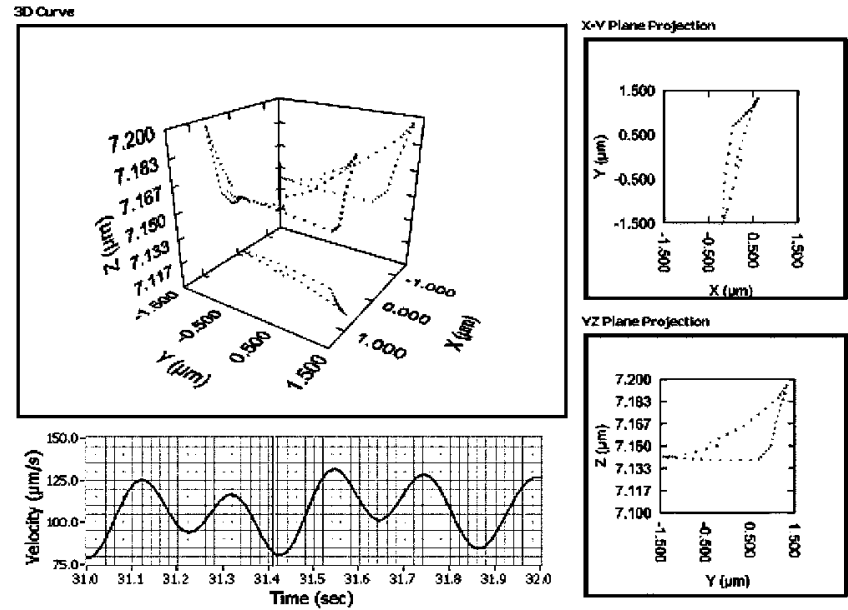

FIG. 8. Cilia tracking with the 3DFM. A bead is fixed to the end(s) of one or more beating cilia. The spatial plots show tracking of the cilia during one complete stroke. The beat extends over $3 \mu \mathrm{m}$, and appears to lie nearly in the $y z$ plane. The velocity waveform to the left of the cursor in the temporal plot corresponds to the spatial plots.

ports (Costar, Cambridge, MA), as described by Matsui et $a l .{ }^{13}$ Cultures were deemed ready for use when evidence of cilia motion or "hurricanes" were visible with a $10 \times$ objective on a phase contrast microscope. The ciliated cultures were washed with phosphate buffered saline (PBS), and then the apical surface was treated with $10 \mathrm{mM}$ dithiothreitol in PBS for 5 min. Gentle washing of the cultures with three changes of PBS was used to remove the mucus layer. The apical surface was then treated with a 1/1000-1/10 000 dilution of biotinylated wheatgerm agglutinin in PBS (Vector labs, Burlingame, CA), for $10 \mathrm{~min}$, and washed three times with PBS. Streptavidin-coated superparamagnetic polystyrene microspheres of either 0.9 (Spherotech, Inc. Libertyville, IL) or $1.0 \mu \mathrm{m}$ diameter (Dynal Biotech, Oslo, Norway) were added undiluted to the apical surface of the cultures and pipetted many times onto the centers of the cultures. Cultures were then incubated at $37^{\circ} \mathrm{C}$ for $10 \mathrm{~min}$, and beads were pipetted again onto the centers, then washed gently once with PBS.

The culture and membrane were then cut from their plastic support and gently cut into fourths with a fresh razor blade and scalpel. Each fourth was then slid gently onto an $18 \times 18 \mathrm{~mm}$ glass coverslip [number 1, Fisher Premium (Fisher Scientific, Pittsburg, PA)], with the apical side up. Approximately $10 \mu \mathrm{L}$ Airway medium and Hepes-buffered airway medium ( $25 \mathrm{~mm}, \mathrm{pH} 7.5)$ were added to each specimen. A second coverslip was placed over the first, using a border of two layers of double sided tape (3M Corporation, St. Paul, MN) to create the specimen chamber. The cilia continued to beat vigorously for at least an hour in these chambers, and often for several hours.

\section{Results}

We attached magnetic beads to the cilia, and engaged our tracking system on the moving beads. A real-time 3D trajectory of a bead attached to the cilia was measured, the plot of which is shown in Fig. 8. The bead size of $1 \mu \mathrm{m}$ and the spacing between cilia being less than $300 \mathrm{~nm}$ precludes

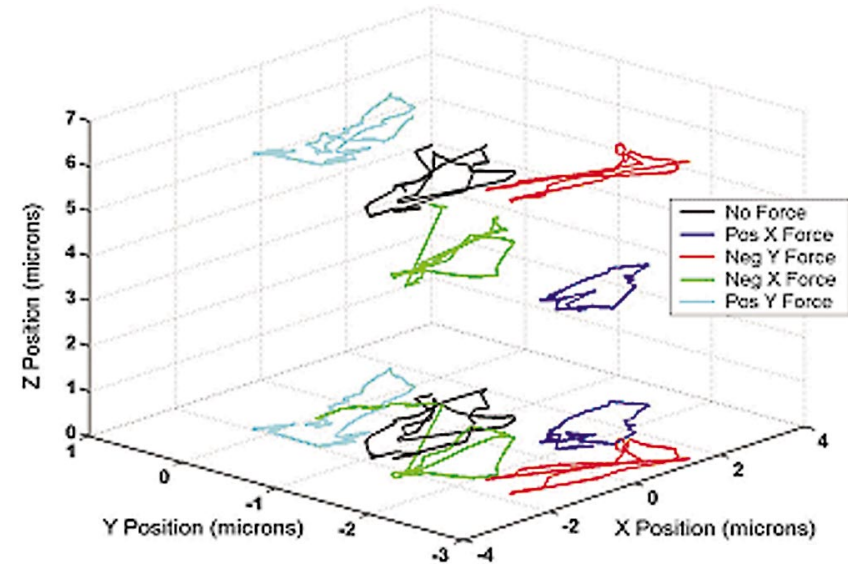

FIG. 9. The trajectories of approximately two cilia beats during the application of force in each of the tetrahedral directions of our pole tips. Projections of the trajectories onto the $X-Y$ plane are shown at the bottom. The directions in which the cilia were pulled correspond to the tetrahedral directions of the pole tips. The poles designated as "Neg Y" and "Pos Y" are above the specimen chamber, whereas those designated as "Neg $X$ " and "Pos X" are below.

our being able to definitively rule out multiple attachments at this time. A stroke length of $3 \mu \mathrm{m}$ was measured for the trajectory of the cilia beat. Our tracking system is capable of measuring the beat cycle in full 3D, significantly including the $z$ motion of the cilia tip. This vertical component of motion is understood to be a critical factor in the operation of the cilia-mucus system as it allows the cilia to retract into the less viscous lower layer during the retraction stroke. During the power stroke, the cilia are at their greatest extension, allowing them to couple more effectively to the more viscous overlying mucus. This $z$ motion is observed in our 3D traces.

We applied forces to the magnetic beads attached to cilia in an initial attempt to explore the response of the cilia to forces. Figure 9 shows that sequential excitation of individual poles results in the average position of the orbit of the cilia being shifted in the direction of the energized pole. Since the poles lie at the corners of a tetrahedron, an energized pole will in general pull a bead both laterally and vertically at the same time. This was observed. The applied magnetic force, estimated to be about $2 \mathrm{pN}$, appears to be enough to shift the beat trajectory significantly without stalling the cilia. This is consistent with the measurements performed on sperm flagella where a stall force of about $250 \mathrm{pN}$ was measured. ${ }^{62}$

\section{Directional magnetic force degeneracy}

To define a 3D coordinate system requires at least four noncoplanar points: one for the origin, and one to define a magnitude and direction for each of the coordinates. Therefore, at least four noncoplanar magnetic pole tip locations are necessary for a true 3DFM. For considerations previously discussed, we chose an equilateral tetrahedron to determine four such points, and defined our origin as the center of the tetrahedron. Although this allows us to pull the bead in various directions in $3 \mathrm{D}$, the range of reachable force angles corresponding to the domain of possible pole excitations does not cover the entire $4 \pi$ sr of the solid angle. 


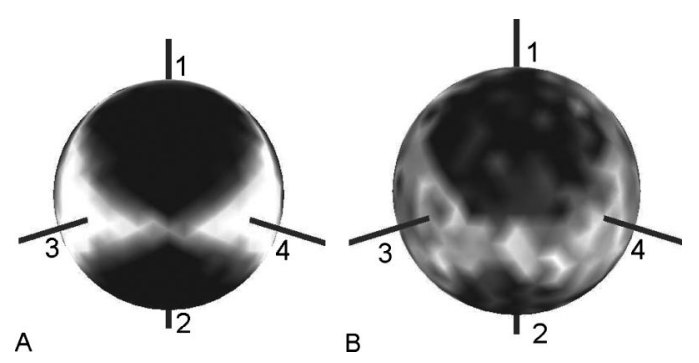

FIG. 10. Spherical plots of forces. Plot A is of a magnetic monopole model, while plot B is of experimental data. The four solid lines projecting from the spheres show directions to the magnetic poles. In both cases a uniform random distribution of normalized pole excitations were applied, and the resulting forces averaged over sampling bins. The averages of the bins are plotted as brightness. Dark regions indicate that no set of coil currents generated force in those directions. Both model and experimental data show that forces can be generated in the directions of the poles and along the lines joining the poles, but not in directions opposite the poles.

To establish the available force directions of the tetrahedral geometry, we conducted the following simulation and subsequently verified it experimentally on the 3DFM. A large ensemble of neutralized, normalized pole excitations were randomly generated and the resulting forces calculated using the monopole model previously described. Neutralized means the pole strengths sum to zero. This ensures that the flux circulating in the iron frame all goes through the pole tips. Without this constraint, "unbalanced" currents would cause additional flux to circulate the iron frame without going through the pole tips. Normalized means the sum of the positive (equal to the sum of the negative) pole strengths is unity. Since the force direction is dependent only on the relative values of the coil currents, normalization will have no effect on the obtainable force directions. A plot of the simulation is shown in Fig. 10(a) where the brightness is proportional to the average of the forces that occurred in that direction. An experimental confirmation of the simulation was performed by applying a randomly generated set of coil currents with the same neutrality and normalization conditions that were used in the simulation. These data are shown in Fig. 10(b). These spherical plots show that forces can be generated in approximately triangular patches about the poles and on lines between poles, but not in directions opposite the poles.

To approximate access to a full 3D force space, we have implemented a control program which cyclically pulls the bead toward different poles with strengths and durations such that the time average of the bead force is the magnitude and direction desired. This method produces a trajectory that is a zig-zag path with step directions determined by the pole locations. The step size would be determined by the viscosity of the specimen and the forces obtained at the sample position, with temporal widths determined by the bandwidth of the magnetics system. As an example of this method, we estimate the motion of a $4.5 \mu \mathrm{m}$ bead seeking the largest "effective" force in an otherwise forbidden direction. For this instrument, in the specimen location of the cilia experiment, with the sample presumed to be the viscosity of water, we would obtain step sizes of approximately $130 \mathrm{~nm}$, with temporal widths of about $0.1 \mathrm{~s}$. This is limited by our current magnetics bandwidth of about $10 \mathrm{~Hz}$. As a result, the time multiplexing approach is not generally applicable to high sensitivity experiments but is useful for bead positioning.

\section{E. Potential improvements to the system}

The system described here is low frequency, essentially a dc prototype with a number of engineering compromises made in the interest of achieving proof of concept. A few improvements of this prototype may be worth consideration, but our attention is better turned toward the development of a second generation system capable of addressing a broader range of scientific applications.

The coils generate sufficient heat at high drive currents that it would be beneficial to add a cooling mechanism, especially if large forces are going to be applied to beads over an extended period of time. Haber and Wirtz $\mathrm{z}^{35}$ accomplished this by winding the wire coils on water cooled brass boxes.

Our tracking noise floor is compromised by a noise bandwidth substantially in excess of the QPD sampling rate. A faster A/D data acquisition board or addition of anti-alias filters between the QPD transimpedance amplifiers and the A/D would provide immediate improvement of tracking resolution.

A significant benefit would be obtained by implementing a 3DFM design as added components to a commercially available microscope. Acceptance of the additional function of the 3DFM by the practicing microscopy community will likely depend on a familiar "look and feel," more easily achieved using an off-the-shelf microscope platform, without sacrifice of such important features as high NA, and the various operational modes such as confocal, DIC, etc., ordinarily available on these platforms. Accordingly, future developments should address high NA systems, e.g., water immersion, on a commercial platform. This substantially decreases the solid angle available for the magnetic system. It would also be desirable to increase the magnetic drive frequency to broaden the range of possible science as well as to improve the tracking feedback loop. A system with these improvements is under development in our laboratory. Another potentially important improvement could be to replace the active $x y z$ feedback stage with dynamically controlled objective focusing and beam deflection in the infinity corrected path of the microscope. This would have the dual benefits of not shaking the biological specimen with high accelerations, while also removing the feedback stage from the already congested volume surrounding the specimen chamber.

\section{ACKNOWLEDGMENTS}

This work was supported by the National Institutes of Health, including Grant Nos. P41-EB02025-21 and R01EB000761.

${ }^{1}$ T. P. Stossel, Science 260, 1086 (1993).

${ }^{2}$ C. L. Rieder and E. D. Salmon, J. Cell Biol. 124, 223 (1994).

${ }^{3}$ L. P. Cramer, T. J. Mitchison, and J. A. Theriot, Curr. Opin. Cell Biol. 6, 82 (1994).

${ }^{4}$ V. Mermall, P. L. Post, and M. S. Mooseker, Science 279, 527 (1998).

${ }^{5}$ J. M. Scholey, I. Brust-Mascher, and A. Mogilner, Nature (London) 422, 746 (2003).

${ }^{6}$ S. A. Endow, BioEssays 25, 1212 (2003). 
${ }^{7}$ P. Huitorel, Biol. Cell 63, 249 (1988).

${ }^{8}$ M. J. Davey et al., Nat. Rev. Mol. Cell Biol. 3, 826 (2002).

${ }^{9}$ V. Ellison and B. Stillman, Cell 106, 655 (2001).

${ }^{10}$ K. S. Wilson and H. F. Noller, Cell 92, 337 (1998).

${ }^{11}$ D. Signor and J. M. Scholey, Essays Biochem. 35, 89 (2000).

${ }^{12} \mathrm{~W}$. Wintermeyer and M. V. Rodnina, Essays Biochem. 35, 117 (2000).

${ }^{13} \mathrm{H}$. Matsui et al., J. Clin. Invest. 102, 1125 (1998).

${ }^{14}$ R. B. Nicklas, J. Cell Biol. 97, 542 (1983).

${ }^{15}$ K. A. Schmitz, D. L. Holcomb-Wygle, D. J. Oberski, and C. B. Lindemann, Biophys. J. 79, 468 (2000).

${ }^{16}$ A. Kishino and T. Yanagida, Nature (London) 334, 74 (1988).

${ }^{17}$ R. V. Skibbens and E. D. Salmon, Exp. Cell Res. 235, 314 (1997).

${ }^{18}$ P. C. Braga and D. Ricci, in Methods in Molecular Biology (Humana Press, Totowa, New Jersey, 2003), Vol. 242.

${ }^{19}$ A. A. Baker et al., Biophys. J. 79, 1139 (2000).

${ }^{20}$ E.-L. Florin et al., Appl. Phys. A: Mater. Sci. Process. 66, S75 (1998).

${ }^{21}$ E. Evans, Annu. Rev. Biophys. Biomol. Struct. 30, 105 (2001).

${ }^{22}$ A. Ashkin et al., Opt. Lett. 11, 288 (1986).

${ }^{23}$ S. M. Block, D. F. Blair, and H. C. Berg, Nature (London) 338, 514 (1989).

${ }^{24}$ M. J. Lang and S. M. Block, Am. J. Phys. 71, 201 (2003).

${ }^{25} \mathrm{H}$. Yin et al., Science 270, 1653 (1995).

${ }^{26}$ J. W. Dai and M. P. Sheetz, Biophys. J. 68, 988 (1995).

${ }^{27}$ E. Hirakawa, H. Higuchi, and Y. Y. Toyoshima, Proc. Natl. Acad. Sci. U.S.A. 97, $2533(2000)$

${ }^{28}$ S. M. Block, L. S. Goldstein, and B. J. Schnapp, Nature (London) 348, 348 (1990).

${ }^{29}$ S. C. Kuo and M. P. Sheetz, Science 269, 232 (1993).

${ }^{30}$ K. Visscher, M. J. Schnitzer, and S. M. Block, Nature (London) 400, 184 (1999).

${ }^{31}$ M. Rief et al., Proc. Natl. Acad. Sci. U.S.A. 97, 9482 (2000).

${ }^{32}$ E. J. G. Peterman, F. Gittes, and C. F. Schmidt, Biophys. J. 84, 1308 (2003).

${ }^{33}$ P. N. Prasad, Opt. Lett. 28, 2288 (2003).
${ }^{34}$ F. H. C. Crick and A. F. W. Hughes, Exp. Cell Res., Suppl. 1, 37 (1949).

${ }^{35}$ C. Haber and D. Wirtz, Rev. Sci. Instrum. 71, 4561 (2000).

${ }^{36}$ F. Ziemann, J. Radler, and E. Sackmann, Biophys. J. 66, 2210 (1994).

${ }^{37}$ F. Assi et al., J. Appl. Phys. 92, 5584 (2002).

${ }^{38}$ M. Barbic et al., Appl. Phys. Lett. 79, 1897 (2001).

${ }^{39}$ P. A. Valberg and D. F. Albertini, J. Cell Biol. 101, 130 (1985).

${ }^{40}$ T. R. Strick et al., Science 271, 1835 (1996).

${ }^{41}$ F. Amblard et al., Rev. Sci. Instrum. 67, 818 (1996).

${ }^{42}$ H. Huang et al., Biophys. J. 82, 2211 (2002).

${ }^{43}$ C. Gosse and V. Croquette, Biophys. J. 82, 3314 (2002).

${ }^{44}$ W. Denk and W. W. Webb, Appl. Opt. 29, 2382 (1994).

${ }^{45}$ M. W. Allersma et al., Biophys. J. 74, 1074 (1998).

${ }^{46}$ A. Pralle et al., Microsc. Res. Tech. 44, 378 (1999).

${ }^{47}$ W. Mark et al., in Computer Graphics: Proceedings of SIGGRAPH'96, 1996 (unpublished), p. 447.

${ }^{48}$ Y. Harada et al., in Laser Tweezers in Cell Biology, edited by M. P. Sheetz (Academic, New York, 1998), Vol. 55, p. 99.

${ }^{49}$ A. Pralle et al., Appl. Phys. A: Mater. Sci. Process. 66, S71 (1998).

${ }^{50}$ A. S. f. T. a. Materials, Annual Book of Standards (1996).

${ }^{51}$ L. Vicci, Department of Computer Science, University of North Carolina, Chapel Hill (2001), p. TR01-31.

${ }^{52}$ F. Gittes and C. F. Schmidt, Opt. Lett. 23, 7 (1998).

${ }^{53}$ H. D. Huang et al., Biophys. J. 82, 2008 (2002).

${ }^{54}$ B. G. Hosu et al., Rev. Sci. Instrum. 74, 4158 (2003).

${ }^{55}$ R. A. Wallace, J. L. King, and G. P. Scott, Biology: The Science of Life (Foresman and Co., Glenview, IL, 1981).

${ }^{56}$ B. K. Rubin, Respir. Care 23, 761 (2002).

${ }^{57}$ R. C. Boucher, Eur. Respir. J. 23, 146 (2004).

${ }^{58}$ M. J. Sanderson and M. A. Sleigh, J. Cell. Sci. 47, 331 (1981).

${ }^{59}$ M. Rautiainen et al., Acta Oto-Laryngol. 112, 845 (1992).

${ }^{60}$ P. F. M. Teunis and H. Machemer, Biophys. J. 67, 381 (1994).

${ }^{61}$ M. A. Chilvers and C. O'Callaghan, Thorax 55, 314 (2000).

${ }^{62}$ M. J. Moritz, K. A. Schmitz, and C. B. Lindemann, Biophys. J. 79, 468 (2000). 